\title{
El carbonato de calcio y sus implicancias en el análisis de conjuntos arqueofaunísticos. El caso Laguna El Doce (departamento General López, provincia de Santa Fe)
}

RMA

Dossier

\author{
Jimena Cornaglia Fernández*
}

\begin{abstract}
*CONICET-Centro de Estudios Interdisciplinarios en Antropología, Facultad de Humanidades y Artes, Universidad Nacional de Rosario, Argentina. E-mail. jimenacornaglia@hotmail.com
\end{abstract}

\begin{abstract}
Resumen
Es frecuente que, bajo determinadas condiciones, se depositen concreciones de carbonato de calcio ( $\left.\mathrm{CaCO}_{3}\right)$ sobre las superficies óseas de los huesos recuperados en sitios arqueológicos. En el presente trabajo se expone el análisis de los efectos tafonómicos de una muestra de especímenes óseos faunísticos procedentes del sitio arqueológico Laguna El Doce, sin limpiar y tras la remoción del carbonato de calcio, con el objeto de evaluar cómo influye la depositación de $\mathrm{CaCO}_{3}$ en el relevamiento de variables antrópicas y naturales, y ver en qué medida puede incrementar - o no- las observaciones de dichas variables. Para este trabajo se seleccionaron los taxones más representados en el conjunto y que registraron evidencias de haber sido ingresados antrópicamente al sitio [venado (Ozotoceros bezoarticus), ñandú (Rhea americana), guanaco (Lama guanicoe) y Artiodactyla; NISP total=1814]. Los resultados obtenidos permitieron observar un incremento significativo en la frecuencia de agentes tafonómicos naturales (i.e. acción de roedores y raíces) y antrópicos, además de la observación de otras variables de interés para el análisis arqueofaunístico como la meteorización, las depositaciones de manganeso, entre otras.
\end{abstract}

Palabras clave: Registro arqueofaunístico; Tafonomía;depositación de $\mathrm{CaCO}_{3}$; variables antrópicas; variables naturales.

The calcium carbonate and its implications for the analysis of archaeological faunal samples. The Laguna El Doce case (General López department, Santa Fe province)

\begin{abstract}
Often, under certain conditions, concretions of calcium carbonate $\left(\mathrm{CaCO}_{3}\right)$ appear on the surface of bone specimens recovered from archaeological sites. In order to assess how the deposition of $\mathrm{CaCO}_{3}$ influences the identification of anthropogenic and natural variables, and to see how far this can increase-or not- the observations of these variables, this paper presents an analysis of the taphonomic effects on a faunal sample from the archaeological site Laguna El Doce after the removal of calcium carbonate. The most represented taxa in the assemblage and those which contained evidence of anthropic modifications were selected [pampas deer (Ozotoceros bezoarticus), rhea (Rhea americana), guanaco (Lama guanicoe) and Artiodactyla, total NISP=1814]. The results obtained allow observing a significant increase in the frequency of natural (i.e. rodents and root etching) and anthropic taphonomic agents, as well as the presence of other variables of interest such as weathering, manganese deposits, among others.
\end{abstract}

Keywords: Archaeofaunistical record, Taphonomy, $\mathrm{CaCO}_{3}$ deposition, Anthropic variables, Natural variables.

En el sitio arqueológico Laguna El Doce (LED), ubicado en el departamento General López de la provincia de Santa Fe, se ha llevado a cabo el estudio de los restos arqueofaunísticos cuyos resultados demuestran que la mayoría de las superficies óseas de los especímenes presentan depositaciones minerales de carbonato de calcio $\left(\mathrm{CaCO}_{3}\right)$ (Cornaglia Fernández 2009, 2011). Si bien el $\mathrm{CaCO}_{3}$ ha favorecido la preservación de las superficies corticales de los especímenes óseos, también ha impedido la observación de ciertas variables tafonómicas antrópicas y naturales de importancia para el análisis del conjunto.

En este trabajo se da a conocer la metodología de análisis empleada para el relevamiento y clasificación de las depositaciones de $\mathrm{CaCO}_{3}$ sobre las superficies óseas basado en cinco estadios cuantitativos y la técnica que se aplicó para su remoción. Por otro lado, se presentan los resultados del análisis macroscópico de los efectos tafonómicos -entendiendo por los mismos a los derivados de la acción de agentes antrópicos y naturales (Lyman 1994)- en los especímenes óseos sin limpiar y tras la remoción del carbonato de calcio, con el objeto de evaluar cómo influyen las depositaciones de $\mathrm{CaCO}_{3}$ en el relevamiento de variables antrópicas y naturales y estimar en qué medida pueden incrementar -o no- las observaciones de estas variables tras la limpieza de los 
especímenes óseos. A su vez, se evalúan estadísticamente las diferencias entre las observaciones de los procesos naturales y antrópicos pre y post limpieza y cómo los resultados obtenidos en esta submuestra pueden proyectarse en toda la muestra de LED.

Teniendo en cuenta lo desarrollado previamente, el presente trabajo tiene como objetivo: 1) relevar cuantitativamente las depositaciones de $\mathrm{CaCO}_{3}$ y aplicar una técnica de remoción del $\mathrm{CaCO}_{3} ; 2$ ) determinar en qué grado pueden estar incidiendo en la observación de variables naturales y culturales las depositaciones de carbonato de calcio; y 3) realizar la interpretación tafonómica de los restos óseos faunísticos del sitio luego de relevar las observaciones de las variables tafonómicas tras la limpieza.

\section{La Laguna el Doce}

\section{Localización y características generales}

La laguna El Doce es un cuerpo de agua permanente ubicado a los $33^{\circ} 54^{\prime} 20^{\prime \prime} \mathrm{S}$ - 62 $02^{\circ} 43^{\prime \prime O}$ a $112 \mathrm{msnm}$, cuya superficie es de 4,32 km² aproximadamente, con una extensión de 2,7 km de largo y 1,6 km de ancho para el año 2006 (Ayuso et al. 2007). El sitio arqueológico LED se halla ubicado en la margen noreste de dicha laguna, al suroeste de la ciudad de Venado Tuerto y a $13 \mathrm{~km}$ aproximadamente de la localidad de San Eduardo. Este cuerpo de agua permanente se ubica en el extremo sur de la provincia de Santa Fe, dentro de la región pampeana, en el área de la Pampa de las lagunas santafesinas (Figura 1).

Fitogeográficamente, LED se encuentra en el Distrito Pampeano Occidental de la provincia Pampeana (Cabrera 1976) y zoogeográficamente pertenece al Dominio Pampásico de la Subregión Guayano-Brasileña (Ringuelet 1955).

La laguna El Doce se origina a partir de una antigua hoyada de deflación excavada durante el último período

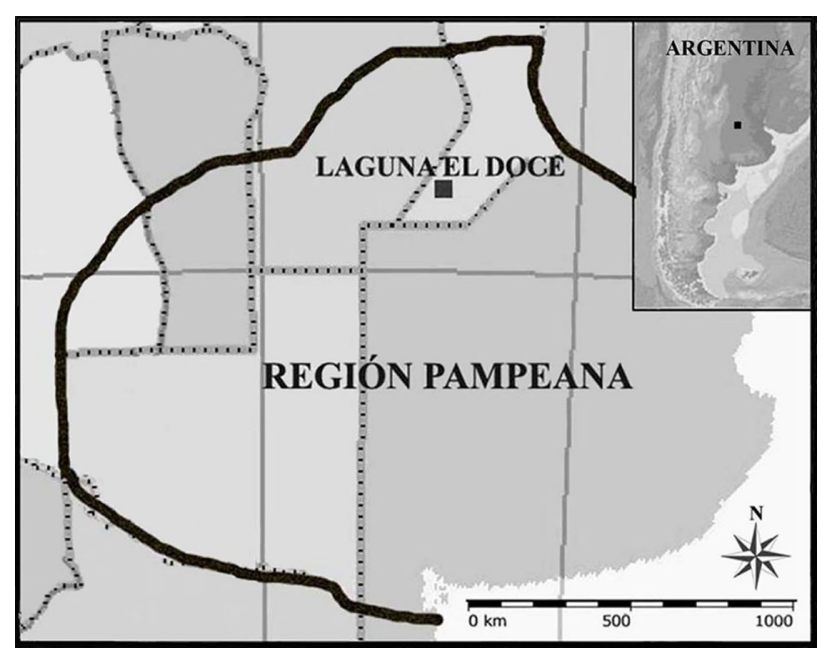

Figura 1. La Región Pampeana y ubicación de LED.

Figure 1. The Pampean Region and location of LED.

climático árido, donde además se formaron dunas eólicas en sus márgenes (Iriondo 1987). Estas dunas hoy conforman topográficamente el sector más alto de este ambiente lagunar y es donde se ubica el mencionado sitio (Figura 2).

\section{Carbonatos, salinidad y el pH del agua y los sedimentos}

Desde el punto de vista edafológico, el área de estudio está compuesta principalmente por sedimentos arenosos de origen eólico, desarrollados durante la última glaciación pleistocénica, depositados durante un período seco, y por sedimentos limosos de origen aluvial mezclados con arenas finas de períodos húmedos (Iriondo 1994). Los suelos presentan distinta proporción de agregados arcillosos y/o de calcretas. Estas últimas se presentan como concreciones y/o láminas de carbonato de calcio que pueden estar presentes de 3 a $4 \%$ y a veces hasta $10 \%$ en suelos arenosos, en cambio, los limos contienen porcentajes inferiores a $1 \%$. Estas acumulaciones, si bien reconocen una variedad de orígenes (procesos sedimentológicos, hidrológicos y

ecológicos; Brown 1997), parecen estar principalmente relacionadas con períodos de aridez, por lo que también pueden utilizarse como indicadores climáticos (Nabel 2011).

Los depósitos cuaternarios donde se emplaza LED, posteriormente fueron retrabajados durante fases áridas sucesivas y sujetos a pedogénesis en períodos húmedos, y descansan de forma erosiva sobre los sedimentos terciarios, constituyentes fundamentales del relleno que forma parte del Mar de Arena Pampeano ("Sistema Eólico

Figura 2. Sitio arqueológico Laguna El Doce. 
Pampeano" sensu Iriondo 1990; Iriondo y Kröhling 2007).

Las aguas de las lagunas del área de estudio presentan distintos tipos de sales y grados de salinidad y alcalinidad, variando desde muy alcalinas o salobres a dulces, dependiendo de las precipitaciones y sequías que diluyen o concentran las substancias químicas y el pH (Catalani 1987).

Actualmente se están realizando estudios sedimentológicos, edafológicos y palinológicos en la laguna El Doce. Los resultados de la medición del pH muestran que los valores más altos se encuentran en el agua de la laguna y en los sedimentos más próximos al pelo de agua con un valor de $\mathrm{pH}$ de 10 (alcalino, pH > $8,2)$, y decrecen con dirección a la barranca, donde los sedimentos tienen un valor de $\mathrm{pH}$ de 8 (salino, $\mathrm{pH}<8,2$ ) (Avila et al. 2010).

\section{Evidencia arqueológica}

LED corresponde a un sitio donde se ha encontrado evidencia de sociedades cazadoras-recolectoras cuya ocupación humana más temprana se ubica en el Holoceno temprano (ca. 8.000 a 7.000 años AP), mientras que su ocupación más reciente corresponde al Holoceno tardío (ca. 2.000 años a 1.000 años AP) (Avila 2011). Las investigaciones arqueológicas realizadas en la misma forman parte de un estudio regional más amplio llevado a cabo en el marco del proyecto de Investigación "Entornos y sociedades. Construcciones de los paisajes arqueológicos en la Pampa de las Lagunas santafesina (Departamento General López, Provincia de Santa $\mathrm{Fe})^{\prime \prime}$ (HUM361, FHUMyAR, UNR). Tales investigaciones comenzaron en el año 2003, cuando se encontraron redepositados en la superficie de la playa y el lecho de la laguna, una gran cantidad de restos arqueológicos pertenecientes principalmente a fauna, restos óseos humanos, material lítico y tiestos cerámicos (Ayuso et al. 2005; Figura 3). En el transcurso de los años se han llevado a cabo recolecciones superficiales sistemáticas, prospecciones, sondeos y, actualmente, se están realizando excavaciones sistemáticas en la barranca contigua a la playa de la laguna.

En el caso específico de los estudios arqueofaunísticos en LED, los mismos consistieron en el análisis de aspectos taxonómicos y tafonómicos del total de los materiales pertenecientes a las diez primeras unidades de recolección trazadas en el año 2003 (Cornaglia Fernández 2009, 2011), y de la evidencia de artefactos y tecnología ósea encontrada en el sitio (Cornaglia Fernández y Buc 2011).

\section{Materiales y Métodos}

Las depositaciones de carbonato de calcio halladas sobre las superficies de los huesos o en el interior de los canales medulares se vinculan a las propiedades de los sedimentos depositados inmediatamente alrededor del material óseo. Es decir, con la génesis y el tipo de sedimento del que se trate y la cantidad de carbonato de calcio que contenga el sustrato. La proporción de $\mathrm{CaCO}_{3}$ puede variar en el tiempo por diversos procesos como la lixiviación, la pedogénesis, y por procesos diagenéticos que tienen que ver con las oscilaciones del nivel freático de la laguna que habrían intervenido en la significativa precipitación de carbonato sobre los huesos (Daniels 1981).

Como fue mencionado anteriormente, los restos óseos faunísticos encontrados en LED presentan en la mayoría de sus superficies óseas precipitaciones de carbonato. Con el objetivo de ahondar en el estudio de dichas depositaciones de $\mathrm{CaCO}_{3^{\prime}}$ se realizó una evaluación cuantitativa del grado de cobertura de la depositación de las concreciones de $\mathrm{CaCO}_{3}$ sobre las superficies corticales siguiendo una escala porcentual con cinco intervalos: 0 (0 $\%) ; 1$ (0-25 \%); 2 (25-50 \%); 3 (50-75 \%); 4 (75-100 \%) (Figura 4), que comprenden las variaciones en la intensidad de las depositaciones desde una superficie no afectada por el $\mathrm{CaCO}_{3}$ (grado 0) hasta la cobertura total del hueso cortical del elemento (grado 4) (y canal medular en huesos largos). Este modo de registro retoma lo desarrollado

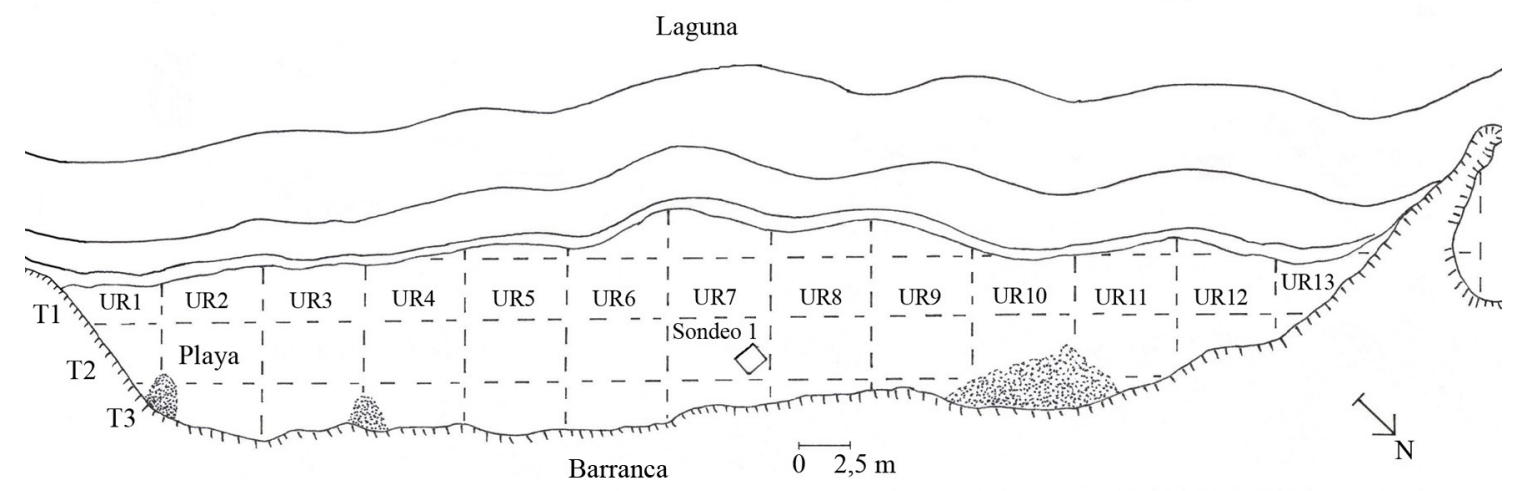

Figura 3. Planta del sitio del año 2003 donde se observan las transectas (T) y unidades de recolección (UR) (modificado de Avila s/f).

Figure 3. Site floor in 2003 where the transects (T) and the collection units (UR) are shown (modified from Avila s/f). 
por Gutiérrez (2004) con una ligera modificación en sus categorías y en la representación gráfica más operativa de la metodología para su relevamiento (Figura 4). ${ }^{1}$

Esta metodología se presenta como una herramienta útil a la hora de registrar la presencia/ausencia de $\mathrm{CaCO}_{3}$, ya que permite, además, apreciar el porcentaje de cobertura de las depositaciones de carbonato de calcio sobre los especímenes óseos, permitiendo evaluar en qué grado se encuentran las superficies óseas comprometidas para la visibilidad de las variables tafonómicas. Esto resulta importante al intentar definir el estado de integridad macroscópica que tienen los especímenes analizados y la influencia que tiene el $\mathrm{CaCO}_{3}$ en la observación de los efectos tafonómicos.

Otra experiencia de registro de las precipitaciones de carbonato de calcio es el trabajo sobre restos óseos humanos desarrollado por González (2007), en el cual realizó un examen del mismo de acuerdo con el aspecto de su manifestación, describiendo la presencia del carbonato en el elemento mediante las categorías: fina película pulverulenta, pequeñas concreciones, cubierta gruesa uniforme y las combinaciones de ellas que se manifestaron; su distribución, detallándola como uniforme o heterogénea $y$, finalmente, la visibilidad que éste permitía de la superficie ósea (buena, regular o mala). Esta metodología de trabajo se refiere más a aspectos cualitativos, y en nuestro caso el interés radica en cuantificar la extensión máxima alcanzada por el carbonato en la superficie del hueso, por eso se aplicó la metodología modificada de Gutiérrez (2004) que permite medir o cuantificar la visibilidad de las variables tafonómicas sobre los huesos.

El material faunístico de LED, perteneciente al total de las dieciocho unidades de recolección realizadas en el año 2003, está compuesto por 9445 especímenes, donde se identificaron 32 taxones, de los cuales 24 se determinaron a nivel de género y especie, y el resto fueron asignados a categorías taxonómicas mayores. Para este trabajo se analizó una sub-muestra conformada por un NISP de 1814 provenientes de las diez primeras unidades de recolección. Se incluyeron todos los especímenes de los taxones más representados en la muestra que registraron evidencias de haber sido ingresados antrópicamente al sitio [guanaco (Lama guanicoe, NISP=1108; NISP\% 61,08\%), venado (Ozotoceros bezoarticus; NISP= 9; NISP\% 0,50), ñandú (Rhea americana; NISP=57; NISP\% 3,14 \%) y Artiodactyla $(\mathrm{NISP}=640 ; \mathrm{NISP} \% 35,28 \%)]$. La submuestra de guanaco analizada representa el 37,48 \% del total de la muestra, la de venado el 33,33\%, la de ñandú el 46,34 \% y la de Artiodactyla el $28 \%$ (Tabla 1).

\footnotetext{
${ }^{1}$ La misma metodología de registro del $\mathrm{CaCO} 3$-pero en una escala porcentual continua- fue aplicada por Ayuso et al. (2006) y Gabrielloni (2009) para el análisis de los restos humanos de LED, llegando a similares resultados que en el análisis del carbonato en restos faunísticos.
}

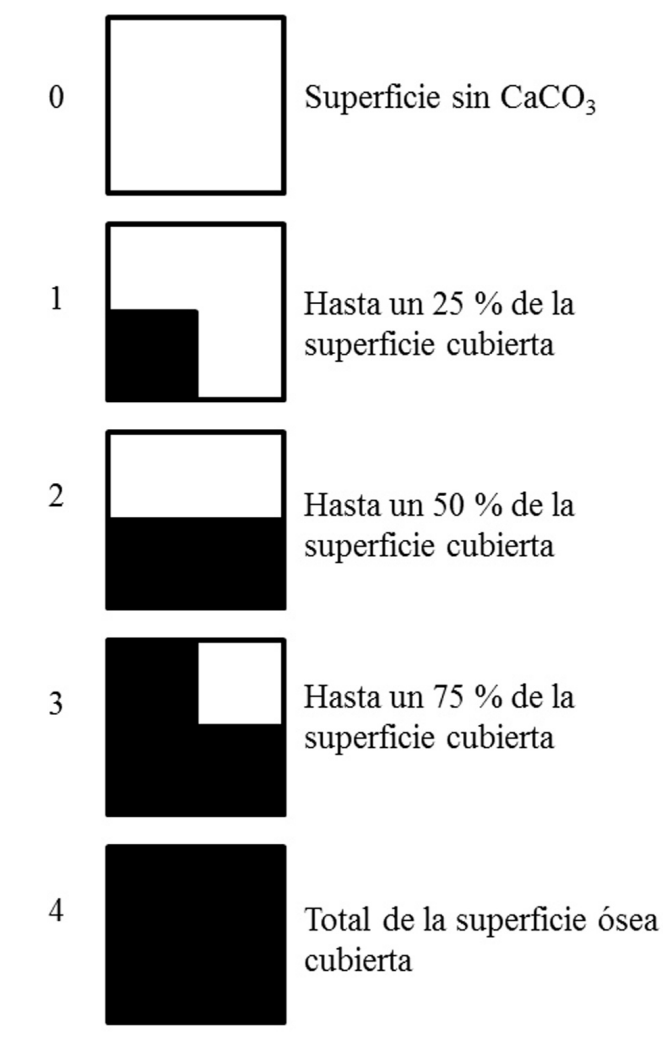

Figura 4. Estadios de cobertura deCaCO sobre restos óseos [0 (0 \%); 1 (0-25\%); 2 (25-50\%); 3 (50-75\%); 4 (75-100\%)].

Figure 4. Coverage stages of $\mathrm{CaCO}_{3}$ on the bones [0 (0\%); $1(0-25 \%)$; $2(25-50 \%) ; 3(50-75 \%) ; 4(75-100 \%)]$.

El trabajo incluyó la remoción de los carbonatos mediante la limpieza manual, y en las muestras en que se dificultó la eliminación mecánica de las incrustaciones o adherencias de $\mathrm{CaCO}_{3}$, las mismas fueron removidas mediante la inmersión en ácido acético diluido al $5 \%$. En otros casos, si existieron pequeñas cantidades aisladas de matriz, se les aplicó el ácido localmente con un cepillo o gotero. Pasados los tres a cinco minutos (o cuando el burbujeo finalizó) se los retiró y se procedió a enjuagarlos minuciosamente en agua destilada y con ayuda de un cepillo suave se eliminaron todos los restos de ácido que pudieron haber quedado.

Cabe aclarar que este método de limpieza, que involucra el empleo de un ácido débil (acético), es un método rápido, que emplea un disolvente económico que no afecta la superficie de los huesos y lo convierte en un tratamiento seguro. Además no influye en los posteriores estudios y análisis que se realicen sobre los huesos (i.e. análisis de isótopos, fechado por carbón radioactivo; Carbonari com. pers. 2010).

Luego de la limpieza, se procedió nuevamente con el análisis macroscópico de las variables antrópicas (presencia/ ausencia de huellas de corte y de termoalteración) y naturales tales como el estado de meteorización (sensu Behrensmeyer 1978), la presencia/ausencia de marcas de roedores, de carnívoros, de raíces y de manchas de 


\begin{tabular}{|c|c|c|c|c|c|}
\hline \multirow[t]{2}{*}{$\begin{array}{c}\text { Taxones } \\
\text { seleccionados }\end{array}$} & \multicolumn{2}{|c|}{$\begin{array}{c}\text { NISP } \\
\text { UR 1-18 }\end{array}$} & \multicolumn{2}{|c|}{$\begin{array}{c}\text { NISP } \\
\text { submuestra } \\
\text { UR 1-10 }\end{array}$} & \multirow{2}{*}{$\begin{array}{c}\text { Proporción de } \\
\text { la submuestra } \\
\text { analizada en el } \\
\text { total de la muestra } \\
\%\end{array}$} \\
\hline & $\mathbf{n}$ & $\%$ & $\mathbf{n}$ & $\%$ & \\
\hline Artiodactyla & 2286 & 42,4 & 640 & 35,28 & 28 \\
\hline Lama guanicoe & 2956 & 54,82 & 1108 & 61,08 & 37,48 \\
\hline $\begin{array}{l}\text { Ozotoceros } \\
\text { bezoarticus }\end{array}$ & 27 & 0,5 & 9 & 0,5 & 33,33 \\
\hline Rhea americana & 123 & 2,28 & 57 & 3,14 & 46,34 \\
\hline Total & 5392 & 100 & 1814 & 100 & \\
\hline
\end{tabular}

ambiental, no es menor respecto de aquella que concierne al análisis arqueofaunístico, ya que la visibilidad de la mayoría de las variables tanto antrópicas como naturales de interés, se encontraron condicionadas por la mayor o menor proporción de superficie carbonatada de los especímenes arqueofaunísticos analizados.

Se considera que el $\mathrm{CaCO}_{3}$ subestimó las reales frecuencias de las diferentes variables de interés como las huellas, y/o la meteorización. Esto se debe a que la mayoría de los especímenes de la muestra registraron diferentes estadios de cobertura de carbonato, que fueron desde un

Tabla 1. NISP de la muestra total y de la submuestra. grado inferior al $25 \%$ de la superficie cortical cubierta (grado $1=28,83 \%$ ), hasta el incremento de la misma evidenciada por un grado $2=21,22 \%$ y $3=17,20 \%$, hasta un alto porcentaje de especímenes que manifestaron un $100 \%$ de sus superficies óseas con depositaciones de carbonato de calcio ( $4=28,22 \%$; Figura 6$)$.

doxido de manganeso (Blumenschine et al. 1996; Lyma 1994; Marean 1995; Mengoni Goñalons 1999, 2006; Olsen y Shipman 1988).

Con los resultados obtenidos del análisis de los especímenes luego de la remoción del $\mathrm{CaCO}_{3}$ se realizó un test estadístico ( $t$ de Student) utilizando el programa PAST 2.14, para determinar la existencia de diferencias estadísticamente significativas entre los resultados del análisis pre y post limpieza del carbonato de calcio, considerándose como tal un valor de $\mathrm{P} \leq 0,05$.

\section{Resultados}

\section{Observaciones tafonómicas realizadas en especímenes} $\mathrm{con} \mathrm{CaCO}_{3}$

Las depositaciones de carbonato de calcio $\left(\mathrm{CaCO}_{3}\right)$ se encuentran presentes en el 95,48 \% de los huesos de la sub-muestra analizada (Figura 5 y 6). Teniendo en cuenta ésto, es importante mencionar que la carbonatación de la superficie cortical es un proceso recurrente en ambientes lagunares pampeanos y, en este caso, se registraron con mayor frecuencia en los huesos que fueron colectados más próximos al cuerpo de agua. Su implicancia

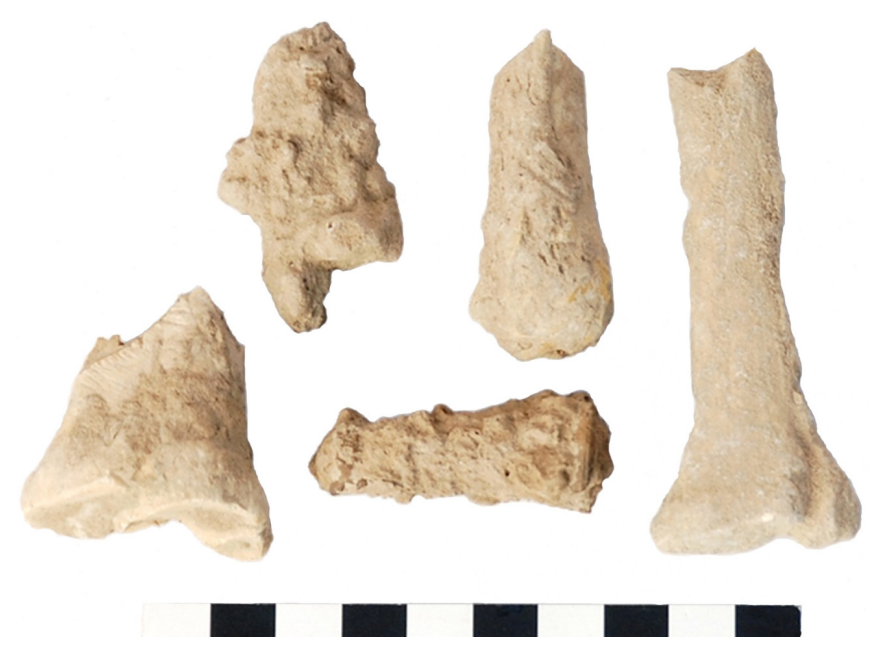

En lo que respecta a la precipitación de óxido de manganeso $\left(\mathrm{MnO}_{2}\right)$, esta variable se reveló con frecuencias mucho menores que el carbonato de calcio ( $n=370 ; 20,40$ $\%$ del total analizado; Tabla 2). En la mayoría de los casos, se presentó en forma de pequeñas manchas difusas y/o pintas oscuras con distribución irregular. La presencia de las mismas está vinculada a ambientes depositacionales húmedos, palustres y/o fluviales (Gifford 1981). Esto se correlaciona con las condiciones del contexto y matriz sedimentaria del sitio (ver supra).

Los resultados sobre las modificaciones de los huesos producto de la actividad de roedores presentaron valores considerables $(n=650 ; 35,83 \%$; Tabla 2$)$. En el sitio se han recuperado restos de mamíferos cavadores como nutria falsa (Myocastor coypus), peludo (Chaetophractus villosus), mulita (Dasypus hybridus), y tuco-tuco (Ctenomys sp.), entre otros. A su vez, en la zona adyacente a la barranca de la laguna, se han detectado cuevas y galerías. Por esta razón, la actividad fosorial de estos mamíferos pudo haber desplazado parte de los materiales, principalmente los de menor tamaño. La migración de materiales provocada por estos roedores también pudo ser favorecida por la reducción del tamaño de los especímenes asociada a la fragmentación ósea registrada en el sitio $(91,51 \%$ del total de los restos analizados) (Cornaglia Fernández 2009, 2011).

Por otro lado, las marcas de carnívoros se registraron en una muy baja frecuencia $(n=17 ; 0,94 \%$ del total de la muestra; Tabla 2) y las marcas de raíces se manifestaron con una frecuencia moderada ( $n=339 ; 18,69 \%$; Tabla 2 ).

\footnotetext{
Figura 5. Especímenes con concreciones de $\mathrm{CaCO}_{3}$ en un grado 3 y 4.

Figure 5. Specimens with concentrations of $\mathrm{CaCO}_{3}$ in grades 3 and 4.
} 


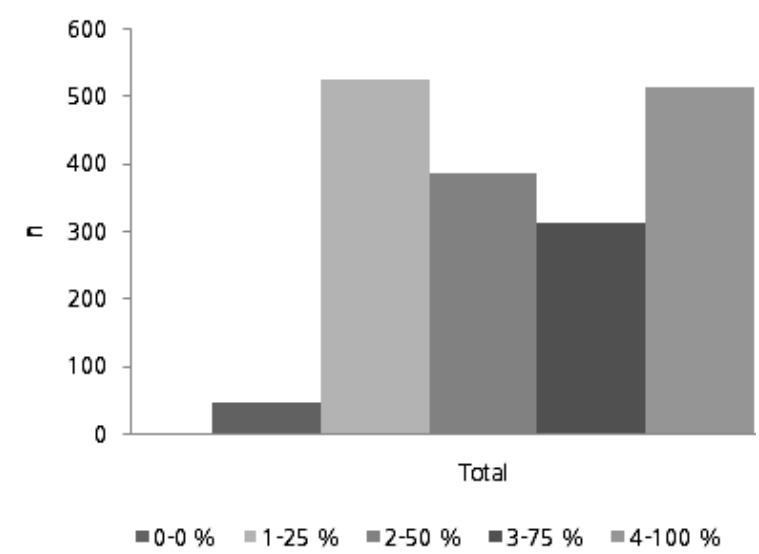

Figura 6. Depositaciones de $\mathrm{CaCO}_{3}$.

Figure 6. Deposits of $\mathrm{CaCO}_{3}$.

Los estadios de meteorización se registraron siguiendo la metodología propuesta por Behrensmeyer (1978), clasificando las modificaciones corticales en seis estadios (0 a 5), y considerando la categoría de indeterminados para aquellos especímenes en los que no se pudo registrar esta variable $(41,41 \%$ de los elementos). En general, la meteorización en el sitio resultó ser baja a moderada, ya que entre los especímenes en que pudo relevarse la variable (58,59\%), una elevada proporción de mamíferos evidenciaron elementos con estadios $0(23,92$ $\%)$, estadio $1(19,46 \%)$ y estadio $2(9,70 \%)$, y en una menor frecuencia se registraron el estadio $3(4,24 \%)$ y $4(1,32 \%)$ (Figura 7$)$.

Una de las causas que probablemente haya favorecido la preservación de las superficies corticales de esta muestra de superficie - de la que se desconoce el tiempo de exposición y/o los eventuales procesos de redepositación y desentierro- de los efectos de la meteorización, puede relacionarse con la elevada prevalencia de carbonato de calcio sobre las superficies óseas (Figura 6), ya que se observó en reiteradas ocasiones que cuando se desprendía alguna capa de $\mathrm{CaCO}_{3^{\prime}}$ la superficie cortical expuesta no presentaba signos de meteorización.

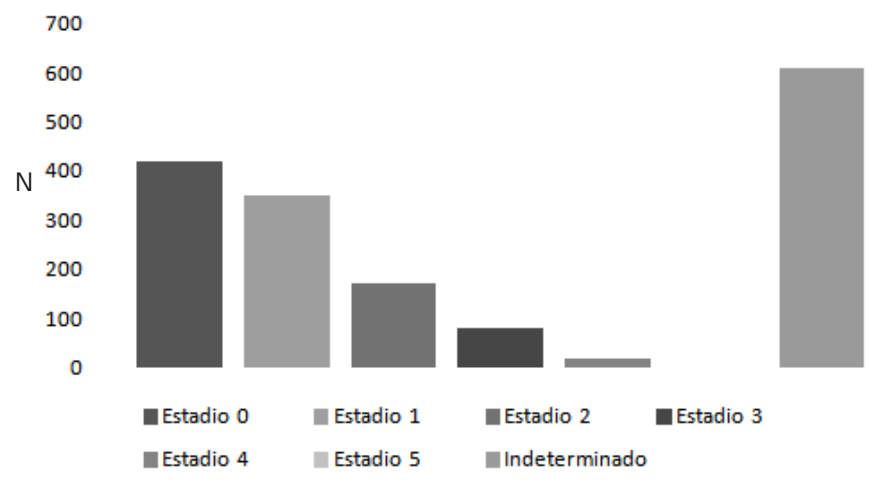

\begin{tabular}{cccccc}
\hline & \multirow{2}{*}{$\begin{array}{c}\text { Variables } \\
\text { tafonómicas }\end{array}$} & \multicolumn{2}{c}{ Con $\mathrm{CaCO}_{3}$} & \multicolumn{2}{c}{$\mathrm{Sin} \mathrm{CaCO}_{3}$} \\
& Carnívoros & 17 & 0,94 & 29 & 1,6 \\
\hline \multirow{2}{*}{$\begin{array}{c}\text { Variables } \\
\text { naturales }\end{array}$} & Roedores & 650 & 35,83 & 839 & 46,25 \\
& Raíces & 339 & 18,69 & 443 & 24,42 \\
& $\mathrm{MnO}_{2}$ & 370 & 20,4 & 720 & 39,69 \\
Variables & Termoalteración & 74 & 4,08 & 96 & 5,29 \\
culturales & Huellas de corte & 204 & 11,25 & 340 & 18,74 \\
\hline
\end{tabular}

Tabla 2. Frecuencias de marcas de carnívoros, roedores, raíces, manchas de manganeso $\left(\mathrm{MnO}_{2}\right)$, evidencias de termoalteración y huellas de corte antes y después de la limpieza del $\mathrm{CaCO}_{3}$.

Table 2. Frequency of carnivore marks, rodents, root etching, manganese stains $\left(\mathrm{MnO}_{2}\right)$, evidence of thermal alteration and cut marks after cleaning of $\mathrm{CaCO}_{3}$.

Por último, se observa que las huellas de corte, marcas vinculadas al procesamiento de las carcasas para su consumo, se presentaron en el 11,25\% del total de los especímenes analizados (Tabla 2). Las alteraciones térmicas se registraron con una frecuencia muy baja ( $n=74 ; 4,08 \%$; Tabla 2). Los especímenes quemados pertenecen en su mayoría a falanges, huesos largos, vértebras, costillas y elementos craneales de Lama guanicoe, así como a falanges y tarsometatarsos de Rhea americana. Las evidencias de combustión en estos huesos apuntan al uso del fuego y/o cenizas en la práctica de preparación de los alimentos. Esto se refuerza a partir de la presencia de otras modificaciones antrópicas (i.e. huellas y/o fracturas) en algunos de los especímenes quemados (Cornaglia Fernández 2009, 2011).

\section{Observaciones tafonómicas realizadas en especímenes $\sin \mathrm{CaCO}_{3}$}

En relación con el análisis realizado luego de la remoción del $\mathrm{CaCO}_{3}$ (Figura 8) se observa que las marcas de roedores (evidenciadas por las modificaciones producto del roído de estos animales) tuvieron mayor influencia que la

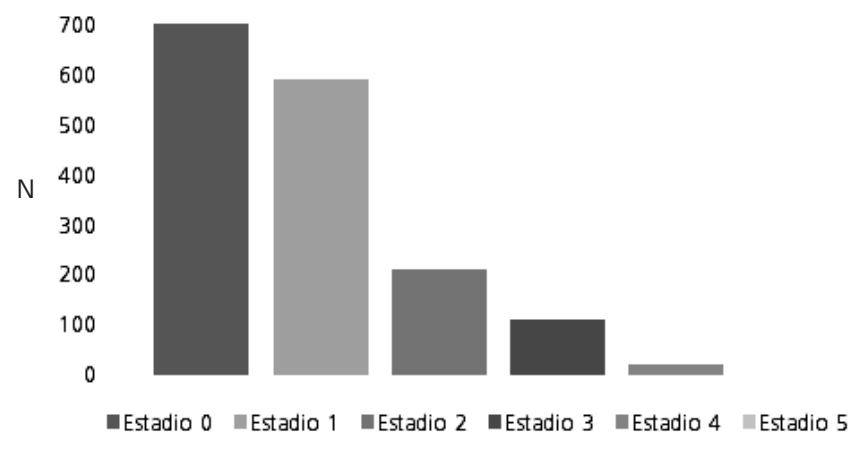

Figura 7. Meteorización antes y después de la limpieza del $\mathrm{CaCO}_{3}$ (sensu Behrensmeyer 1978).

Figure 7. Weathering before and after cleaning of $\mathrm{CaCO}_{3}$ (sensu Behrensmeyer 1978). 
observada anteriormente sobre el registro faunístico $(\mathrm{n}=$ 839; 46,25 \%; Tabla 2). Esto se vincula a la información tafonómica generada en otros sitios arqueológicos a nivel regional, donde se observa una activa influencia perturbadora de los roedores (Bonomo y Massigoge 2004; Gutiérrez 2004; Politis y Madrid 1988; Salemme 1987; entre otros).

Las marcas producidas por carnívoros siguen siendo aún escasas ( $n=29 ; 1,60 \%$; Tabla 2 ), y la mayoría se presentaron en las epífisis de húmero de Lama guanicoe y en las epífisis de tarsometatarso y tibiatarso de Rhea americana.

El grabado dendrítico producto de la actividad de raíces ( $n=443 ; 24,42 \%$; Tabla 2$)$ y la tinción por manganeso ( $n=720 ; 39,69 \%$; Tabla 2) son también modificaciones corticales que aumentaron su frecuencia en el análisis del registro arqueofaunístico. La influencia del óxido de manganeso podría deberse a las oscilaciones de la napa freática o a los avances estacionales de la laguna, momento en que los huesos quedarían sumergidos en el agua.

En lo que respecta a la meteorización, los resultados demuestran que el perfil cambia luego de la eliminación del $\mathrm{CaCO}_{3}$. El grado de meteorización no es acentuado en el sitio, ya que se observa que la mayoría de los especímenes no exhiben indicios de meteorización $(41,66 \%)$, y los que la presentan, evidencian signos bajos (estadio 1= 34,95 \%; estadio 2=14,24\%). Mientras que las alteraciones más severas atribuibles a la meteorización en un estadio 3 y 4, tienen una representación menor (estadio 3= 7,06 \%; estadio 4= 2,08) (Figura 7).

A diferencia de lo observado antes de la limpieza del $\mathrm{CaCO}_{3}$, el perfil de meteorización sugiere que gran parte de los restos no estuvieron expuestos por un tiempo largo a las condiciones atmosféricas, esto es evidenciado por los estadios bajos de meteorización, luego de la remoción del $\mathrm{CaCO}_{3}$ (Figura 7). Esto se relaciona con la observación reiterada durante el estudio del conjunto óseo, que cuando se despegaba alguna capa de carbonato, la
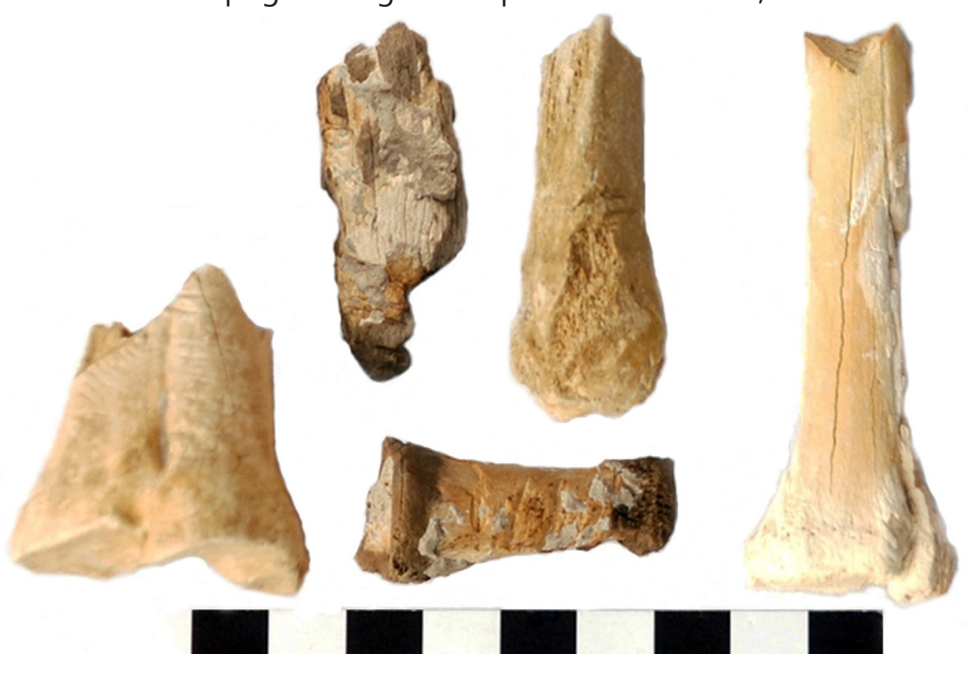

superficie del elemento expuesta no presentaba rastros de meteorización.

Otros efectos tafonómicos vinculados a la acción antrópica, como las huellas de corte, aumentaron en su frecuencia, registrándose en 340 especímenes $(n=340$; 18,74 \%; Tabla 2), en varios elementos de Lama guanicoe (húmero, radiocúbito, tibia, metapodios, falanges, vértebras, pelvis) y en especímenes de Rhea americana (tibiatarso, tarsometatarso, fémur) y Ozotoceros bezoarticus (tibia). Estas marcas están localizadas tanto en las diáfisis como en las epífisis de los huesos largos. Debido a su ubicación y a los elementos en que se presentan, algunas de estas huellas indicarían actividades de desmembramiento y desarticulación de las carcasas (Binford 1981; Mengoni Goñalons 1999).

Las evidencias de termoalteración incrementaron levemente su frecuencia ( $n=96 ; 5,29 \%$; Tabla 2 ) y se presentaron en especímenes de Artiodactyla (astillas y fragmentos de diáfisis de huesos largos), Lama guanicoe (falange, autopodios, vértebras, fémur, tibia, húmero, radiocúbito, metapodios, mandíbula, pelvis) y Rhea americana (falange y metatarso).

Además de la presencia de huellas de corte y alteraciones térmicas que se incrementaron luego de la remoción del carbonato de calcio sobre las superficies corticales de los huesos (Tabla 2), los nuevos datos obtenidos incorporan información nueva y refuerzan lo inferido anteriormente en otros trabajos (Cornaglia Fernández 2009, 2011). De acuerdo con los mismos, las modificaciones predepositacionales permiten hipotetizar que la actividad humana fue el principal agente responsable de la depositación de los huesos que conforman las concentraciones de materiales analizados. Esto también lo demuestra la gran cantidad de restos con fracturas de naturaleza antrópica y la evidencia de tecnología ósea en especímenes de Artiodactyla y de Lama guanicoe (Cornaglia Fernández y Buc 2011).

\section{Discusión y Algunas Conclusiones}

La información obtenida de la comparación entre los huesos analizados con carbonato (Figura 6 y 7; Tabla 2) y luego de su remoción (Figura 7; Tabla 2) permitieron observar un incremento significativo en la frecuencia de agentes tafonómicos naturales (i.e. acción de roedores, raíces) y antrópicos (i.e. huellas de corte, termoalteración), además de otras variables de interés como meteorización y depositaciones de manganeso, entre otras.

Figura 8. Especímenes luego de la limpieza de las concreciones de $\mathrm{CaCO}_{3}$.

Figure 8. Specimens after the cleaning of $\mathrm{CaCO}_{3}$ concentrations. 
Se considera que la evaluación cuantitativa de las precipitaciones carbonatosas antes y después de su remoción, resulta de gran importancia para definir el grado en que los restos óseos fueron afectados por los procesos tafonómicos. De acuerdo con los resultados del análisis, las precipitaciones densas y uniformes de carbonato influyeron en la buena visibilidad de la corteza ósea, provocando la subestimación de las reales frecuencias de diferentes variables de interés (meteorización, marcas de roedores, de raíces, huellas, entre otras).

Luego de la eliminación del $\mathrm{CaCO}_{3}$, las variables naturales, como las marcas de radículas producto de la actividad de raíces se incrementaron en un 5,73 \%, las de roedores en un $10,42 \%$ y las de carnívoros en un 0,66 \%.

Las depositaciones químicas como las manchas de manganeso, son las que acrecentaron su frecuencia en mayor medida (19,29\%). Las tafonómicas, vinculadas a la acción antrópica, tales como las huellas de corte y evidencias de combustión, aumentaron en un 7,49 \% y $1,32 \%$ respectivamente.

A su vez, mediante el análisis estadístico de los datos presentados en el apartado Resultados (ver supra) se observa que las diferencias reconocidas antes y después de la remoción del $\mathrm{CaCO}_{3}$ son estadísticamente significativas, especialmente en la meteorización, el $\mathrm{MnO}_{2}$, las marcas de roedores, las marcas de raíces y las huellas $(\chi 2, P \leq 0,05)$, no así en las marcas de carnívoros $\mathrm{y}$ en las evidencias de combustión $(\chi 2, \mathrm{P} \geq 0,05)$.

Por lo tanto, la remoción en el laboratorio del carbonato de calcio se presenta como una herramienta útil que permite registrar en detalle variables naturales y antrópicas. Los datos obtenidos del análisis de los efectos tafonómicos, basados en una sub-muestra, nos pueden dar una idea de cuán subestimadas están las reales frecuencias de las variables tafonómicas en el conjunto, y debe ser útil e importante a la hora de realizar cualquier inferencia en registros que presentan concreciones de carbonato de calcio.

La submuestra analizada en este trabajo, que representa el 33,64\% de la muestra compuesta por un total de 5392 restos óseos faunísticos (Tabla 1), da cuenta de un incremento en las frecuencias de las variables naturales y culturales, cuando el $\mathrm{CaCO}_{3}$ es eliminado. La selección de la misma no fue aleatoria, sin embargo, consideramos que es representativa del total de la muestra, ya que cubre todos los taxones seleccionados que comprenden más del $50 \%$ del total de las unidades de recolección. Los datos obtenidos del análisis de la misma resultan importantes a la hora de hacer una proyección al total del conjunto óseo compuesto por 18 unidades de recolección, ya que tendríamos un 66,35 \% de la muestra con nulos o diferentes grados de cobertura de carbonato de calcio que podrían potencialmente presentar evidencias de efectos tafonómicos de los cuales no se tiene conocimiento debido al impedimento de su observación por las depositaciones (ver Tabla 3, Figuras 9 y 10).

Como se desprende del análisis, una variedad de agentes y procesos tafonómicos actuaron sobre los restos óseos faunísticos, conduciendo a una historia tafonómica compleja del conjunto arqueofaunístico de LED, evidenciada por una serie de modificaciones tanto naturales como culturales sobre las superficies óseas de la muestra analizada.

Además, es importante mencionar la acción hídrica sobre este conjunto, ya que los materiales analizados para este trabajo forman del material de superficie hallado en el año 2003 sobre la playa y el pelo de agua de la laguna. En este caso la dinámica lagunar causa la erosión retrocedente de la barranca y la re-exposición y traslado de los huesos. Es válido aclarar que el retroceso de la línea de costa de la laguna es un fenómeno reciente desde el punto de vista de una cronología arqueológica, pero es un proceso que participó activamente en la estructuración espacial definitiva y también en la exposición a la acción de determinados procesos naturales y culturales sobre el conjunto óseo faunístico.

Así, en un lapso de tan sólo 10 años la laguna avanzó varios metros sobre el terreno, reflejando la posibilidad de que estos restos faunísticos hayan sido enterrados originalmente en un sector alejado del borde lagunar.

En suma, el sitio LED presenta una compleja historia tafonómica y múltiples procesos tanto de índole natural como cultural han incidido en la formación del registro arqueológico. El empleo de la remoción de $\mathrm{CaCO}_{3}$ se presenta como una herramienta que contribuye a mejorar la observación de variables importantes en lo que concierne al análisis arqueofaunístico, ya que la visibilidad de la mayoría de las variables tanto antrópicas como naturales de interés, se encontraron condicionadas por la mayor o menor proporción de superficie carbonatada de los especímenes arqueofaunísticos investigados.

Los resultados obtenidos permitieron ampliar las inferencias alcanzadas previamente (Cornaglia Fernández 2009, 2011) acerca de los diferentes procesos que participaron en la formación del registro arqueofaunístico de LED. Mediante el análisis de los efectos tafonómicos aplicado en esta investigación y la técnica de remoción de carbonato de calcio se ha logrado incrementar la información relacionada con la integridad de dicho registro. Así, se ha contribuido con la interpretación arqueológica a partir de un nuevo tipo de evidencia.

Finalmente, podemos agregar que pensamos aplicar la metodología expuesta en este trabajo a los materiales óseos faunísticos provenientes de estratigrafía que se encuentran en proceso de análisis con el objetivo de 


\begin{tabular}{lcccc}
\hline & & \multicolumn{3}{c}{ Proyección } \\
& $\begin{array}{c}\text { Variables } \\
\text { tafonómicas }\end{array}$ & $\begin{array}{c}\text { Submuestra } \\
\text { NISP } \mathbf{1 8 1 4}\end{array}$ & $\begin{array}{c}\text { Muestra } \\
\text { NISP 5392 }\end{array}$ \\
& Carnívoros & 29 & 1,6 & 4,76 \\
\hline Variables & Roedores & 839 & 46,25 & 137,47 \\
naturales & Raíces & 443 & 24,42 & 72,59 \\
& MnO 2 & 720 & 39,69 & 117,08 \\
Variables & Termoalteración & 96 & 5,29 & 15,72 \\
\hline culturales & Huellas de corte & 340 & 18,74 & 55,7 \\
\hline
\end{tabular}

Tabla 3. Proyección de las variables tafonómicas al total de la muestra.

Table 3. Taphonomic variables of the total sample.

obtener nuevos resultados que amplíen la información sobre los distintos agentes naturales y culturales que participaron en la conformación del registro arqueofaunístico procedente de la Laguna El Doce.

Rosario, 15 de Noviembre 2011

\section{Agradecimientos}

A la Dra. Lorena L'Heureux por la lectura crítica del manuscrito. A Yanina Vrankovic' por las fotos. A los evaluadores por sus valiosos comentarios y sugerencias. Este trabajo forma parte del proyecto HUM361 radicado en la Facultad de Humanidades y Artes de la Universidad Nacional de Rosario, y dirigido por Carlos N. Ceruti y Juan David Avila. Los contenidos y opiniones vertidos aquí son de mi absoluta responsabilidad.

\section{Bibliografía}

Avila, J. D. 2011. Resultados de los fechados radiocarbónicos del sitio Laguna El Doce, Departamento General López, Provincia de Santa Fe. Relaciones de la Sociedad Argentina de Antropología 36: 337-343.

Avila, J. D., J. Cornaglia Fernández y C. Gabrielloni. 2010. Informe de la Campaña arqueológica al sitio Laguna El Doce. Departamento General López, Santa Fe. MS.

Ayuso, M. P., J. D. Avila y M. Gallego. 2005. Informe sobre las investigaciones arqueológicas efectuadas en el sitio Laguna El Doce, San Eduardo, Provincia de Santa $\mathrm{Fe}$. Entregado a la Jefatura Comunal de San Eduardo, Departamento General López, Provincia de Santa Fe. MS.

Ayuso, M. P., J. D. Avila, A. Alonso, J. Cornaglia Fernández, C. Gabrielloni, M. Gavilán, C. Píccoli, S. Martínez, R. Moreira, M. Gallego y M. C. Barboza. 2007. Arqueología en el sur de Sta. Fe: Laguna El Doce (Depto. Gral. López, Pcia. de Sta. Fe). Ponencia presentada en el XVI CNAA, Mendoza. MS.

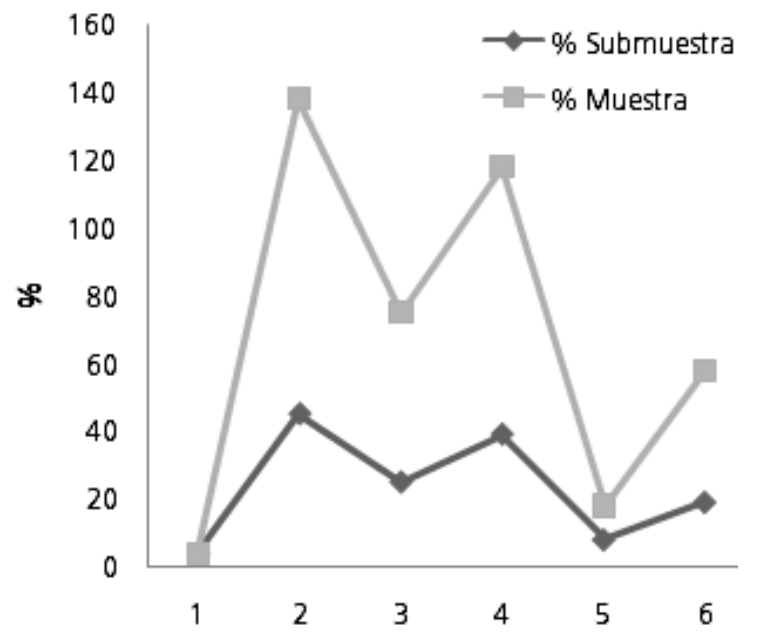

Figura 9. Proyección de las variables tafonómicas [1) marcas de carnívoros; 2) marcas de roedores; 3 ) marcas de raíces; 4) manchas de $\mathrm{MnO}_{2}$; 5) termoalteración y 6) huellas de corte].

Figure 9. Projection of the taphonomic variables [1) carnivore marks; 2) rodent marks; 3) root marks; 4) $\mathrm{MnO}_{2}$ stains; 5) thermal alteration and 6) cut marks].

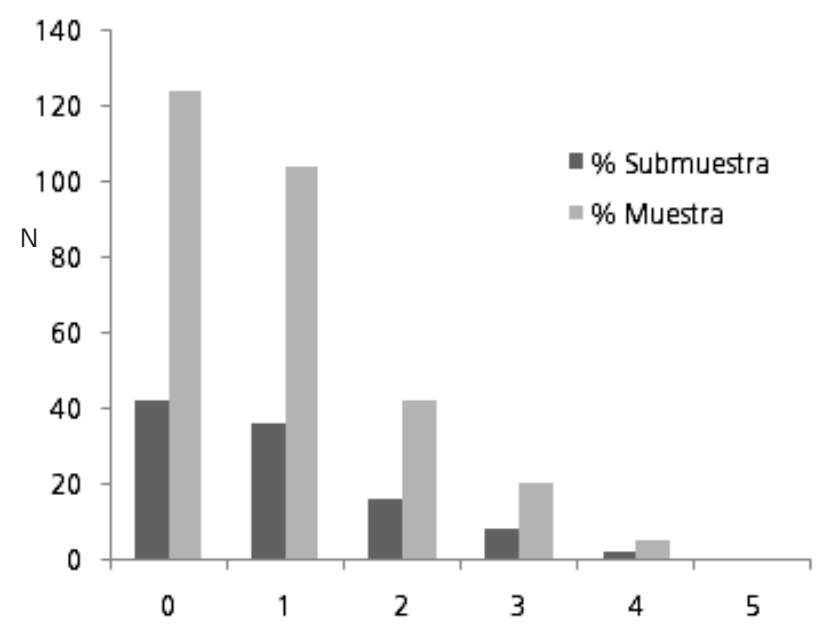

Figura 10. Proyección de la meteorización al total de la muestra (sensu Behrensmeyer 1978).

Figure 10. Weathering of the total sample (sensu Behrensmeyer 1978).

Behrensmeyer, A. K. 1978. Taphonomic and ecology information from bone weathering. Paleobiology 4 (2): 150-162.

Binford, L. R. 1981. Bones: Ancient Men and Modern Myths. Academic Press, Nueva York.

Blumenschine R. J., C. W. Marean y S. D. Capaldo.1996. Blind test of inter-analyst correspondence and accuracy in the identification of cut marks, percussion marks, and carnivore tooth marks on bone surfaces. Journal of Archaeological Science 23: 493-507.

Bonomo, M., A. Massigoge. 2004. Análisis tafonómicos del conjunto faunístico del sitio arqueológico Nutria Mansa 1 (partido de General Alvarado). (Eds.) G. Martínez, 
M. A. Gutiérrez, R. Curtoni, M. Berón y P. Madrid, Aproximaciones Contemporáneas a la Arqueología Pampeana.Perspectivas teóricas, metodológicas, analíticas y casos de estudio, 93-111, Facultad de Ciencias Sociales, UNCPBA, Olavarría.

Brown, A. G. 1997. Alluvial Geoarchaeology. Floodplain Archaeology and Environmental Change. Cambridge University Press, Cambridge.

Cabrera, A. L. 1976 Regiones Fitogeográficas Argentinas. Enciclopedia Argentina de Agricultura y Jardinería 2 (1). Editorial Acme. Buenos Aires.

Catalani, W. R. 1987. Procesos geomorfológicos situados en una parte de la Pampa Hundida que abarca el SW de la Provincia de Santa Fe. Boletín de la Sociedad Argentina de Estudios Geográficos GAEA.: 68-69. Buenos Aires.

Cornaglia Fernández, J. 2009. "Zooarqueología del Holoceno tardío en el sur de Santa Fe. El sitio Laguna El Doce". Facultad de Humanidades y Artes, Universidad Nacional de Rosario, Argentina, pp. 261. Tesis de grado. Rosario.

Cornaglia Fernández, J. 2011. Análisis tafonómico del registro arqueofaunístico de la Pampa de las lagunas santafesinas. El sitio Laguna El Doce (Provincia de Santa Fe, Argentina). (Eds). N. Kuperszmit, L. Mucciolo, T. Lagos Mármol y M. Sacchi, Entre Pasados y Presentes 3. Estudios contemporáneos en Ciencias Antropológicas, Buenos Aires. En prensa.

Cornaglia Fernández, J., N. Buc. 2011. Evidence of bone technology on the Santa Fe's Pampa lagoons. The Laguna El Doce site (Santa Fe Province, Argentina). (Eds). A. Choyke y S. O'Connor, Raw material, Oxbow Books, Oxford. En prensa.

Daniels, V. 1981. Manganese-containing stains on excavated pottery sherds. Masca Journal 1: 230-231.

Gifford, D. P. 1981. Taphonomy and Paleoecology: A Critical Review of Archaeology's Sister Disciplines. (Ed.) M. B. Schiffer, Advances in Archaeological Method and Theory 4: 157-235, Academic Press, Nueva York.

González, M. E. 2007. Estudios de interés tafonómico en los restos óseos humanos de Laguna Tres Reyes 1 (Partido de Adolfo Gonzales Chaves, provincia de Buenos Aires). Intersecciones en Antropología 8: 215-233.

Gutiérrez, M. A. 2004. "Análisis tafonómicos en el Área Interserrana (Provincia de Buenos Aires)". Facultad de Ciencias Naturales y Museo, Universidad Nacional de La Plata. Tesis Doctoral. La Plata.

Iriondo, M. 1987. Geomorfología y Cuaternario de la provincia de Santa Fe (Argentina). D'Orbignyana 4: 1-54.

Iriondo, M. 1990. Map of the South American plains Its present states. (Ed.) Rabassa,J., Quaternary of South America and Antarctic Peninsula7: 197-218., A. A. Balkema, Rotterdam.

Iriondo, M. 1994. Los Climas Cuaternarios de la Región Pampeana. Comunicaciones (Nueva Serie) 4 (2) del Museo Provincial de Ciencias Naturales "Florentino Ameghino" Santa Fe.

Iriondo, M. y D. Kröhling. 2007. Geomorfología y sedimentología de la Cuenca superior del Río Salado (sur de Santa Fe y noroeste de Buenos Aires, Argentina). Latin American Journal of Sedimentology and basin analysis 14 (1): $1-23$

Lyman, R. L. 1994. Vertebrate Taphonomy.Cambridge University Press, Cambridge.

Manzi, R., M. Gallardo.1970.Geografía de Santa Fe. Ed. Spadoni, Mendoza.

Marean, C. W. 1995. Of Taphonomy and Zooarchaeology. Evolutionary Anthropology 4 (2): 64-72.

Mengoni Goñalons, G. 1999. Cazadores de guanacos de la estepa patagónica. SAA, Buenos Aires.

Mengoni Goñalons, G. 2006-2009. Zooarqueología en la práctica: algunos temas metodológicos. Xama 19-23: 83-113. Unidad de Antropología- CRYCYT. Mendoza.

Nabel, P. Subsuelo. Sedimentos Pampeanos. Atlas ambiental de Buenos Aires: http://www.atlasdebuenosaires.gov.ar [Consulta 5 de Octubre de 2011]

Olsen, S. L., P. Shipman.1988. Surface modification on bone: trampling versus butchery. Journal of Archaeological Science 15: 535-553.

Politis, G., P. Madrid. 1988. Un hueso duro de roer: Análisis preliminar de la tafonomía del sitio Laguna Tres Reyes 1 (Pdo. de Adolfo Gonzales Chaves, Pcia. de Buenos Aires). (Ed.) N. Ratto y A. Haber, De Procesos, Contextos y otros Huesos, 29-44, ICA y Facultad de Filosofía y Letras (UBA), Buenos Aires.

Ringuelet, R. 1955. Panorama zoogeográfico de la provincia de Buenos Aires. Notas del Museo de La Plata, 18 (156), Zoología. La Plata.

Salemme, M. C. 1987. "Paleoetnozoología del sector Bonaerense de la Región Pampeana, con especial atención a los mamíferos". Facultad de Ciencias Naturales y Museo, Universidad Nacional de La Plata. Tesis Doctoral. La Plata. 